\title{
Nanometre scale 3D nanomechanical imaging of semiconductor structures from few nm to sub-micrometre depths
}

\author{
${ }^{1 *} \underline{\text { O.V. Kolosov, }}{ }^{2}$ F. Dinelli, ${ }^{1}$ A. Robson, ${ }^{1}$ A. Krier, ${ }^{1}$ M. Hayne, V. I. Fal'ko and ${ }^{3}$ M. Henini \\ ${ }^{1}$ Physics Department, Lancaster University, Lancaster, LA1 4YB, UK, \\ ${ }^{2}$ CNR - INO, Pisa, Italy \\ ${ }^{3}$ School of Physics and Astronomy, The University of Nottingham, Nottingham, UK \\ *e-mail: o.kolosov@lancaster.ac.uk, http: www.nano-science.com
}

Multilayer structures of active semiconductor devices (1), novel memories (2) and semiconductor interconnects are becoming increasingly three-dimensional (3D) with simultaneous decrease of dimensions down to the few nanometres length scale (3). Ability to test and explore these 3D nanostructures with nanoscale resolution is vital for the optimization of their operation and improving manufacturing processes of new semiconductor devices. While electron and scanning probe microscopes (SPMs) can provide necessary lateral resolution, their ability to probe underneath the immediate surface is severely limited. Cross-sectioning of the structures via focused ion beam (FIB) to expose the subsurface areas often introduces multiple artefacts that mask the true features of the hidden structures, negating benefits of such approach. In addition, the few tens of micrometre dimension of FIB cut, make it unusable for the SPM investigation.

Here, we present two complementary advanced characterization methodologies enabling mapping of 3D nanostructure of semiconductor devices, memories and interconnects with nanometre scale resolution. First one is based on the wellknown ability of elastic stress to propagate in the bulk of the material. In order to create such nanoscale stress we use the combination of SPM and ultrasound - Ultrasonic Force Microscopy (UFM) (4) known for its ability to detect subsurface features in the solid state materials (5). UFM is using the standard contact atomic force microscope (AFM) setup where the high frequency (HF) several MHz range, but very small sub-nanometre amplitude ultrasonic vibration is applied to the studied sample. As vibration frequency is much higher than the resonance frequency of AFM tip and cantilever, nanoscale sized apex of the tip dynamically presses the sample, creating the HF oscillating strain field in the subsurface of the sample (6) as shown in Fig.1a. The subsurface features in the material in the volume reached by this strain field result in the modification of the dynamic reaction force acting on the probe tip. The change in the oscillating reaction force is detected as the average "ultrasonic" force due to the force "rectification" at the nonlinear tip-surface contact (4) resulting in a mapping of surface and near-surface properties of the material.

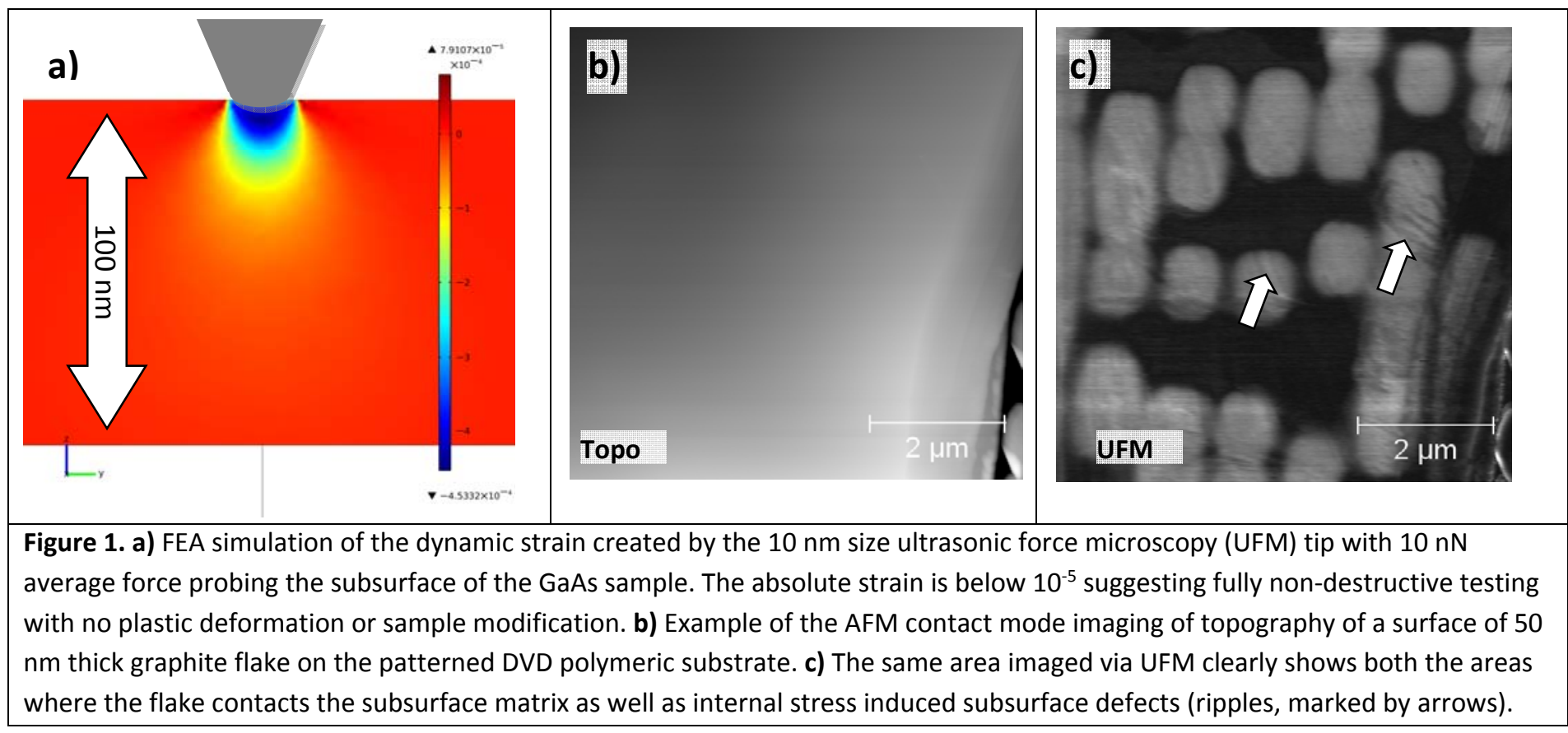

While observing subsurface defects with significant mismatch of mechanical properties have been already reported reported by the authors who pioneered this work (5) and several other groups - including observing voids and delaminations (7-9), vacuoles (10) or inorganic inclusions in the cells (11), the question remains whether nanomechanical mapping can reveal compositional differences in the solid state inorganic materials, a task essential for the semiconductor 
industry, metallization applications and nanotechnology. Here we show that such task is fully feasible and can produce the unambiguous images of internal morphology of iii-v InAs/GaAs semiconductor quantum dot structures under atomically flat GaAs capping layer (Fig. 2).

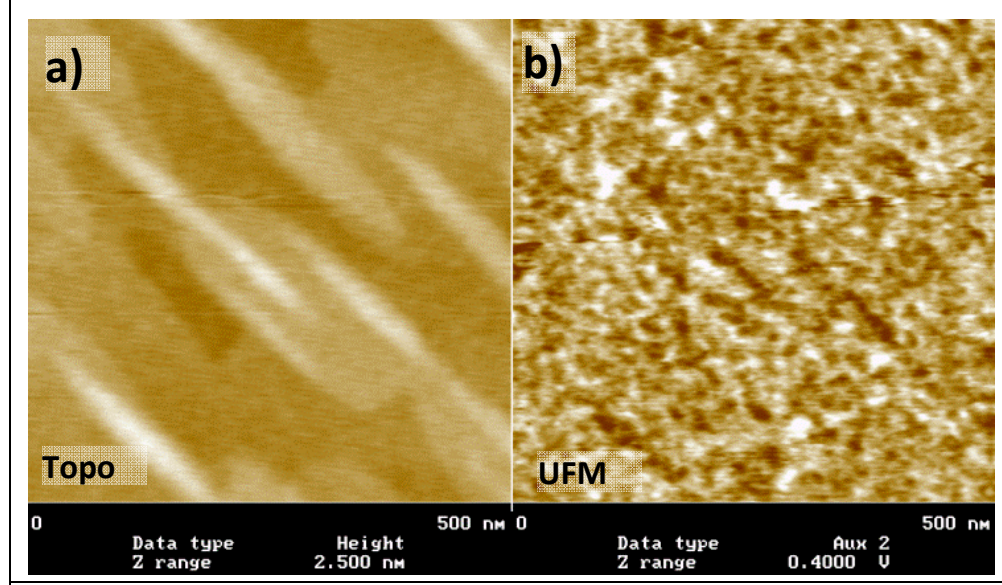

Figure 2. a) AFM topography and b) UFM nanomechanical imaging of of InAs quantum dots fully capped by $50 \AA$ GaAs layer. Both images are obtained simultaneously, with AFM showing only atomically flat terraces, while UFM revealing QD's under the capping layer (image width $500 \mathrm{~nm}$ ). Note that the smallest feature in UFM subsurface image of InAs QD's is 5nm.
One of the key questions still remains - whether the superior subsurface imaging capability of UFM is linked with the acoustic wave scattering from the internal defects as suggested by Dravid et al (9) and other groups $(11,12)$ with subsurface features detected via changes in the amplitude and/or phase of the wave on the sample surface. Alternatively, such subsurface sensitivity can be the result of the elastic field propagating from the tip-surface contact as suggested by Kolosov and Yamanaka (13, 14), Atalar's (15) and other research groups (16). One of the strong reservations against the elastic field scattering hypothesis would be that both the size of the subsurface inclusion, $r \sim 10-50 \mathrm{~nm}$, and the distance from the inclusion to the sample surface $h \sim$ 50-200 $\mathrm{nm}$ are 1,000 to 10,000 times smaller than the ultrasonic wavelength $\lambda \sim 1 \mathrm{~mm}$ (at few MHz). It is well known since the works of Rayleigh that the intensity of the wave scattered from the defect in such case is governed by the $(r / \lambda)^{-4} \approx 10^{-12}$ corresponding to the amplitude of the scattered wave $a \approx \sqrt{10^{-12}} \approx 10^{-6}$ - a vanishing value. At the same time, we noted that in order to correctly analyse the scattered field, one has to account for the near-field stress and strain term in the vicinity of the inclusion - terms that are often neglected in the far field. We use such analysis that have been performed elsewhere for the case of the spherical inclusion in the solid body (17) in application to the nanoscale features, and found that the modulation of the amplitude and the phase of the ultrasonic wave on the sample surface of GaAs sample with the $r=50 \mathrm{~nm}$ InAs inclusion positioned at $h=100 \mathrm{~nm}$ depth is one the order of $2.5 \times 10^{-7}$ and $6 \times 10^{-4} \mathrm{rad}$ (or $0.03^{0}$ ), respectively (Fig. 3) suggesting that scattering should be excluded as the origin of the subsurface contrast. At the same time, the stiffness of elastic contact is modified by the few percent by such inclusion, strongly suggesting that it is elastic field that produces the superior subsurface imaging in the nanomechanical SPM.

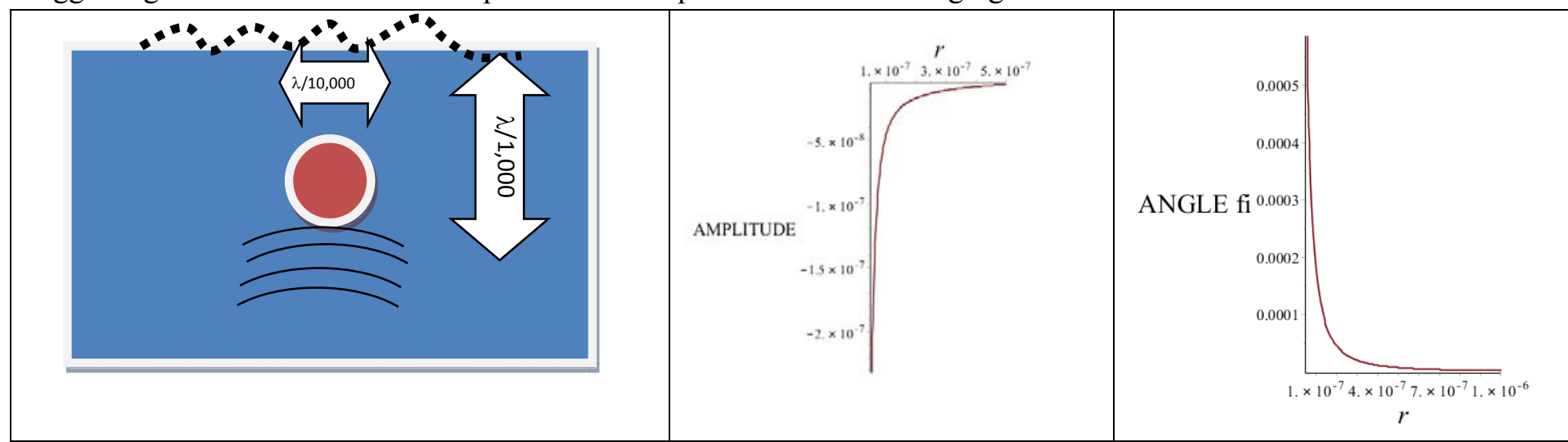

Figure 3. a) Sketch of the inclusion in the sample probed by UFM or other nanomechanical probe - the characteristic inclusion size and depth are compared to the ultrasonic wavelength $\lambda$. Analytical model results of the change in the a) amplitude and $b$ ) phase of the ultrasonic vibration due to the presence of the $r=50 \mathrm{~nm}$ InAs inclusion at the depth $h=100 \mathrm{~nm}$ in the GaAs matrix. Our calculations indicate extremely small variations of the ultrasonic wave, therefore suggesting that it is the elastic strain mechanism as in the Fig. 1 is responsible for the subsurface imaging in the semiconductor nanostructures in the Fig. 2.

The lateral resolution for all subsurface UFM (5) and its follow-up developments (11) is directly determined by the elastic field equations and is on the same order as the depth of subsurface feature (Fig. 1). So for graphite flake that is approximately $50 \mathrm{~nm}$ thick, the transition between supported and suspended areas happens on the order of few tens of $\mathrm{nm}$, whereas finest features of InSb quantum dots observed in UFM through atomically flat terraces of $5 \mathrm{~nm}$ GaAs cap (figure 2f) have corresponding $5 \mathrm{~nm}$ size. 
Finally, in case where the subsurface imaging exceeding the depth of the elastic field is needed, we apply a new method by Kolosov et al $(18,19)$ allowing the nearly atomic flatness via Ar ion polishing of the sample using beam exit geometry (BEXP, Fig. 4). As opposed to the standard beam entrance or FIB cutting, BEXP beam produces a nearly-atomically flat cross-section adjacent to the intact original surface, therefore allowing unimpeded 3D exploration of semiconductor nanostructures at the depths from $\sim 5 \mathrm{~nm}$ to $\sim 1$ um using SPM or scanning electron microscopy.

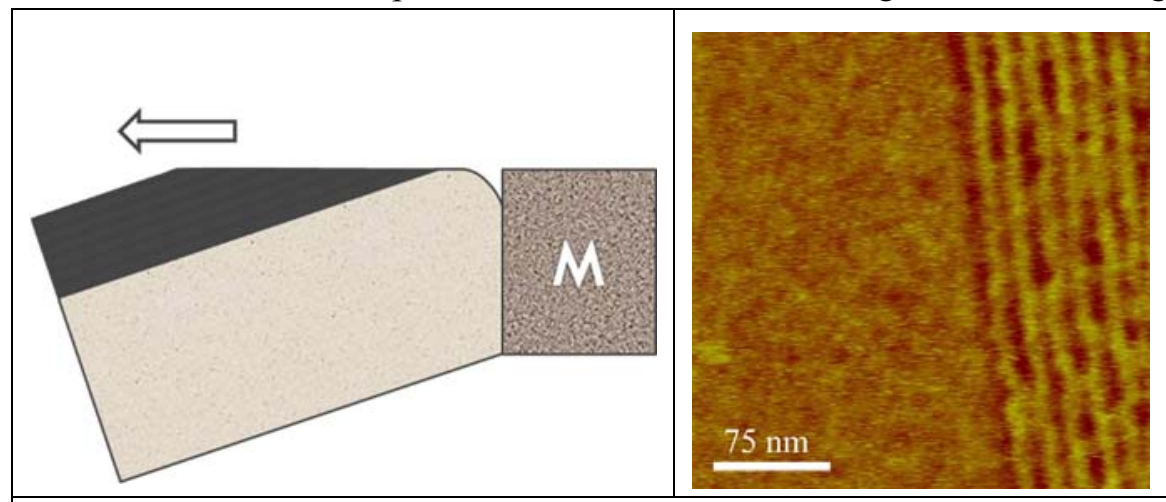

Fig. 4. a) Schematics of the Beam Exit Cross-sectional polishing (BEXP) using Ar ions. The nearly atomic flatness of the cut immediately adjacent to the intact sample surface allows 3D exploration of semiconductor nanostructures at the depths from $\sim 5 \mathrm{~nm}$ to $\sim 1 \mu \mathrm{m}$. b) Nanomechanical UFM image showing a 2-nm AlAs/GaAs superlattice. AlAs layers, appearing darker than GaAs layers, distinguished through oxide growth. The layers appear much thicker than $2 \mathrm{~nm}$ in the image because the cross-section is performed at a shallow angle with respect to the sample surface. As such, the $75 \mathrm{~nm}$ scale bar corresponds to $15 \mathrm{~nm}$ in the growth direction. Note that features inside each layer are clearly visible - the performance not achievable by the TEM cross-section averaging the properties of the layers.

(Ulis). 2013:102-5.

2. Meena JS, Sze SM, Chand U, Tseng T-Y. Overview of emerging nonvolatile memory technologies. Nanoscale Research Letters. 2014;9.

3. Lu W, Lieber CM. Nanoelectronics from the bottom up. Nat Mater. 2007;6(11):841-50.

4. Kolosov O, Yamanaka K. Nonlinear detection of ultrasonic vibrations in an atomic-force microscope. Japanese Journal of Applied Physics Part 2-Letters. 1993;32(8A):L1095-L8.

5. Yamanaka K, Ogiso H, Kolosov O. Ultrasonic force microscopy for nanometre resolution subsurface imaging. Applied Physics Letters. 1994;64(2):178-80.

6. Fischer-Cripps AC. Introduction to Contact Mechanics. Ling FF, editor. N.Y, USA: Springer; 2007. 221 p.

7. Geer RE, Kolosov OV, Briggs GAD, Shekhawat GS. Nanometer-scale mechanical imaging of aluminum damascene interconnect structures in a low-dielectric-constant polymer. Journal of Applied Physics. 2002;91(7):4549-55.

8. McGuigan AP, Huey BD, Briggs GAD, Kolosov OV, Tsukahara Y, Yanaka M. Measurement of debonding in cracked nanocomposite films by ultrasonic force microscopy. Applied Physics Letters. 2002;80(7):1180-2.

9. Shekhawat GS, Dravid VP. Nanoscale imaging of buried structures via scanning near-field ultrasound holography. Science. 2005;310(5745):89-92.

10. Tetard L, Passian A, Thundat T. New modes for subsurface atomic force microscopy through nanomechanical coupling. Nature Nanotechnology. 2010;5(2):105-9.

11. Tetard L, Passian A, Venmar KT, Lynch RM, Voy BH, Shekhawat G, et al. Imaging nanoparticles in cells by nanomechanical holography. Nature Nanotechnology. 2008;3(8):501-5.

12. Verbiest GJ, Oosterkamp TH, Rost MJ. Subsurface-AFM: sensitivity to the heterodyne signal. Nanotechnology. 2013;24(36).

13. Kolosov O. UFM shakes out the details at the nanoscopic scale. Materials World. 1998;6(12):753-4.

14. Yamanaka K, Ogiso $\mathrm{H}$, Kolosov O. Analysis of subsurface imaging and effects of surface elasticity in the ultrasonic force microscope. Jpn J Appl Phys Part 1 - Regul Pap Short Notes Rev Pap. 1994;33(5B):3197-203.

15. Sarioglu AF, Atalar A, Degertekin FL. Modeling the effect of subsurface interface defects on contact stiffness for ultrasonic atomic force microscopy. Applied Physics Letters. 2004;84(26):5368-70.

16. Ebeling D, Eslami B, Solares SD. Visualizing the Subsurface of Soft Matter: Simultaneous Topographical Imaging, Depth Modulation, and Compositional Mapping with Triple Frequency Atomic Force Microscopy. Acs Nano. 2013;7(11):10387-96.

17. Korneev VA, Johnson LR. SCATTERING OF ELASTIC-WAVES BY A SPHERICAL INCLUSION .2. LIMITATIONS OF ASYMPTOTIC SOLUTIONS. Geophys J Int. 1993;115(1):251-63.

18. Kolosov OV, Grishin I, Jones R. Material sensitive scanning probe microscopy of subsurface semiconductor nanostructures via beam exit Ar ion polishing. Nanotechnology. 2011;22(18):185702. Epub 2011/03/19.

19. Robson AJ, Grishin I, Young RJ, Sanchez AM, Kolosov OV, Hayne M. High-Accuracy Analysis of Nanoscale Semiconductor Layers Using Beam-Exit Ar-Ion Polishing and Scanning Probe Microscopy. Acs Applied Materials \& Interfaces. 2013;5(8):3241-5. 